\title{
Predictive value of preoperative weight loss on survival of elderly patients undergoing surgery for esophageal squamous cell carcinoma
}

\author{
Hanlu Zhang", Yushang Yang", Qixin Shang, Xiaoyang Li, Wenping Wang, Yang Hu, Yong Yuan, \\ Yun Wang, Long-Qi Chen \\ Department of Thoracic Surgery, West China Hospital of Sichuan University, Chengdu 610041, China \\ Contributions: (I) Conception and design: LQ Chen, H Zhang, Y Yang; (II) Administrative support: LQ Chen, Y Wang, Y Hu, Y Yuan; (III) \\ Provision of study materials or patients: LQ Chen, Y Wang, Y Hu, Y Yuan; (IV) Collection and assembly of data: W Wang; (V) Data analysis and \\ interpretation: H Zhang, Y Yang; (VI) Manuscript writing: All authors; (VII) Final approval of manuscript: All authors. \\ \#These authors contribute equally to this work. \\ Correspondence to: Long-Qi Chen. Department of Thoracic Surgery, West China Hospital of Sichuan University, Chengdu 610041, China. \\ Email: drchenlq@scu.edu.cn.
}

\begin{abstract}
Background: The role of preoperative weight loss (PWL) has rarely been studied in elderly patients undergoing surgery for esophageal squamous cell carcinoma (ESCC). The aim of this study is to evaluate whether PWL is an important determinant of survival in elderly patients following surgery for ESCC.

Methods: This retrospective study included a total of 974 elderly patients ( $\geq 65$ years) who underwent esophagectomy for ESCC at the West China Hospital, Sichuan University from August 2005 to April 2013. PWL was determined as the percentage weight loss during the 3 months before admission. Patients were divided into three groups according to the PWL, defined as normal $(\mathrm{PWL}=0)$, slight $\mathrm{PWL}(0<\mathrm{PWL}<10 \%)$ and severe PWL (PWL $\geq 10 \%)$. Prognostic factors were evaluated by using univariate and multivariate analyses. Results: Patients were categorized as normal ( $\mathrm{n}=605,62.11 \%)$, slight PWL ( $=284, \mathrm{n}=29.17 \%)$ and severe PWL ( $\mathrm{n}=85,8.73 \%$ ). Compared with patients without PWL, both the patients with slight and severe PWL suffered from higher risk of tumor depth invasion ( $\mathrm{P}=0.002$ and $\mathrm{P}<0.001$, respectively), lymph node metastasis $(\mathrm{P}=0.009$ and $\mathrm{P}=0.004$, respectively) and advanced stage $(\mathrm{P}=0.006$ and $\mathrm{P}<0.001$, respectively). Both patients with slight and severe $\mathrm{PWL}$ were significantly associated with worse overall survival compared with patients without $\mathrm{PWL}(\mathrm{P}=0.036$ and $\mathrm{P}<0.001$, respectively). Multivariate analysis revealed severe $\mathrm{PWL}$ was an independent prognostic factor of overall survival [hazard ratio (HR) $=1.534,95 \% \mathrm{CI}$ : $1.163-2.024$ ] after correcting for sex, body mass index (BMI), T stage, $\mathrm{N}$ stage, grade, and adjuvant therapy.

Conclusions: For elderly patients undergoing surgery for ESCC, PWL provided prognostic value for depth of tumor invasion, lymph node metastasis and advanced stage. PWL $\geq 10 \%$ was an independent predictor of worse overall survival.
\end{abstract}

Keywords: Esophageal neoplasms; esophagectomy; elderly; survival; weight loss

Submitted Jun 10, 2019. Accepted for publication Oct 11, 2019.

doi: $10.21037 /$ tcr.2019.10.29

View this article at: http://dx.doi.org/10.21037/tcr.2019.10.29

\section{Introduction}

ESCC is the predominant histological type of esophageal cancer in Eastern countries (1). Radical surgical resection is the primary curative therapy for patients with ESCC.
Despite improvements in perioperative management, instruments and surgical techniques, prognosis of ESCC remains poor (2). Pathological findings of the tumor such as tumor size, grade of differentiation and presence of lymph 
node metastases, are all prognostic indicators that allow patient stratification and clinical decision-making, but these can only be obtained post-operatively.

A large variation in age occurs in the patients with ESCC. Currently, the elderly population is increasing, which results in an increased number of elderly ESCC patients requiring surgery. Compared to younger patients, elderly patients frequently have one or more comorbidities and are often "frail" (3). There is a need for age-specific studies and preoperative estimations of overall survival for elderly patients, which might enable eligible elderly patient selection for esophagectomy.

Host-related factors, such as malnutrition, are reported to reduce overall survival after gastrointestinal surgery (4). However, there is currently no standard criterion to assess nutritional status in patients with ESCC (5). Due to reduced food intake and increased energy consumption caused by systemic inflammation induced by the tumor, PWL is commonly associated with patients with esophageal cancer $(6,7)$. PWL is a very simple objective preoperative nutritional parameter used to assess the nutritional condition of patients, and has been recognized as an independent predictor of long-term postoperative survival in various cancers, including esophageal carcinoma (8-10). However, whether PWL could provide predictive information on the long-term outcome of elderly patients with ESCC remains uncertain $(8,11)$. We hypothesize that preoperative nutritional assessment based on PWL is a promising indicator for predicting postoperative long-term survival of elderly patients.

\section{Methods}

\section{Patients}

From August 2005 to April 2013, a total of 974 elderly patients undergoing esophagectomy for ESCC in the Department of Thoracic Surgery, West China Hospital, Sichuan University were included in this retrospective study. Although there was no agreement on a definition of elderly, according to the literature $(3,12)$, an age cutoff of $\geq 65$ years was used. All of the patients were preoperatively diagnosed with ESCC by upper gastrointestinal endoscopy and biopsy. Tumors were staged to be resectable according to contrast CT scan of the chest and upper abdomen, esophageal barium swallow and endoscopic ultrasound. In selected cases, integrated fluorodeoxy-glucose positron emission tomography was performed to exclude the presence of metastatic disease. The exclusion criteria were as follows:
(I) patients with non-squamous cell carcinoma; (II) patients with incidental findings of M1 stage during operation; (III) patients receiving neoadjuvant therapy; (IV) patients with incomplete data. This study was approved by West China Hospital of Sichuan University Biomedical Research Ethics Committee [No. 2016049].

The current height and weight of patients were measured before surgery. Stable weight was defined as the weight of three months before the admission provided by the patient. PWL was defined as the percentage weight loss during the 3 months before admission. Even though preoperative weight loss as a negative outcome has been described in many studies, there is currently no standard criterion for PWL. The $10 \%$ weight loss cutoff was chosen in our study because PWL $>10 \%$ was considered to represent severe nutritional risk (13). In the presented study, patients were subsequently classified into three groups: normal, slight PWL $(0<$ PWL $<10 \%)$ and severe PWL (PWL $\geq 10 \%)$. BMI was calculated as "weight $(\mathrm{kg}) /$ height $\left(\mathrm{m}^{2}\right)$ ".

Data were obtained retrospectively, including patient demographics, surgical procedures, treatment details, pathological stage of disease and survival. The primary outcome was overall survival. Covariates included age, sex, BMI, T stage, $\mathrm{N}$ stage, grade, tumor location and adjuvant therapy. Overall survival was the time from surgery to the date of death or last clinic visit. Patients alive at the last follow-up were censored for overall survival.

\section{Surgical procedure}

Patients without distant metastasis or definitive evidence of extensive adjacent organ invasion underwent surgical resection. All patients underwent a curative transthoracic subtotal esophagectomy with two-field lymphadenectomy, including the Sweet, Ivor-Lewis and McKeown approaches. Patients with tumors located at the middle or lower thoracic esophagus with no evidence of lymph node involvement in the superior mediastinum or in the neck received esophagectomy via the Sweet or Ivor-Lewis esophagectomies. Patients with tumors located at the middle or upper thoracic esophagus or with possible lymph node metastasis in the superior mediastinum were operated via the McKeown esophagectomy. The gastroesophageal anastomosis was created in the chest or in the neck, depending on the location of the tumor. Esophago-gastrostomy was created between the proximal esophageal remnant and the gastric conduit using either a circular stapler or a hand-sewn procedure based on surgeons' own technical expertise. 


\section{Follow-up}

All patients were seen in follow-up at our outpatient department every three months in the first two years after resection, and semi-annually thereafter. The follow-up protocol included history taking, physical examination and chest abdominal CT scans. Upper GI endoscopy, radionuclide bone scans, PET-CT scans, and abdominal ultrasound were arranged if clinically indicated. Overall survival was calculated from the date of esophagectomy until death or the end of the follow-up period, which was up to five years post-operatively (14).

\section{Statistical analysis}

Continuous data are presented as the mean and standard deviations. Categorical variables are shown as frequency and percentages. The $t$ and chi-square tests were used for comparison between the groups. The Kaplan-Meier method was used to estimate survival. The log-rank test was employed to compare for survival differences. Univariate analysis was used to examine association between potential predictors and survival. Hazard ratios (HRs) with 95\% confidence intervals (CIs) were utilized to quantify the association between predictors and survival. Univariate factors with a $\mathrm{P}$ value of $<0.25$ and believed to be associated with cancer-related deaths, were entered into a multivariate Cox proportional hazards regression model. Backward stepwise elimination of variables was used to construct the final model. $\mathrm{P}<0.05$ was considered as statistically significant. Data analysis was performed using SPSS software version 24.0 (SPSS Inc., Chicago, IL, USA).

\section{Results}

One thousand and one hundred eighteen elderly patients with ESCC were included in our database. One hundred forty-four Patients were excluded as they did not meet the inclusion criteria. These included 124 patients with nonsquamous cell carcinoma, 8 patients with incidental finding of M1 stage during the operation, 8 patients who had missing data, and 4 patients receiving neoadjuvant therapy. Finally, a total of 974 patients were included in the analysis. The mean follow-up time was 33.9 months.

\section{PWL and clinicopathological characteristics (Table 1)}

The demographic and clinical characteristics of the included
974 patients (787 males and 187 females) are summarized in Table 1. Patients were categorized as three groups, including 605 patients $(62.11 \%$ ) had no PWL, 284 patients (29.17\%) had slight PWL (0-10\%), 85 patients $(8.73 \%)$ had severe PWL (>10\%). A significant higher number of patients presenting with severe PWL were found to have $\mathrm{BMI}<18.5(\mathrm{P}<0.001)$. Patients with slight and severe PWL suffered from higher risk of local tumor invasion $(\mathrm{P}=0.002$ and $\mathrm{P}<0.001$, respectively), lymph node involvement $(\mathrm{P}=0.009$ and $\mathrm{P}=0.004$, respectively) and advanced stage disease $(\mathrm{P}=0.006$ and $\mathrm{P}<0.001$, respectively). There was no significant difference in preoperative comorbidities, tumor differentiation status, tumor location, surgical approach and adjuvant therapy when comparing slight PWL and severe PWL with patients without PWL.

\section{Overall survival}

The one-, three- and five-year overall survival rates were $79.1 \%, 52.4 \%$ and $41.9 \%$ in the normal group, $77.6 \%$, $45.0 \%$ and $32.7 \%$ in the sight PWL group, and $64.9 \%$, $29.4 \%$ and $20.2 \%$ in the severe PWL group, respectively. Kaplan-Meier curves comparing patients of the three groups regarding overall survival showed statistically significantly worse overall survival for patients with slight PWL $(\mathrm{P}=0.036)$ and severe PWL $(\mathrm{P}<0.001)$ compared to patients with no PWL (Figure 1).

\section{Predictive factors for overall survival (Table 2)}

In the univariate analysis (Table 2), sex (hazard ratio 1.388; $\mathrm{P}=0.004$ ), slight $\mathrm{PWL}$ (hazard ratio $1.217 ; \mathrm{P}=0.035$ ), severe PWL (hazard ratio $1.842 ; \mathrm{P}<0.001$ ), $\mathrm{T}$ stage (hazard ratio 2.418; $\mathrm{P}<0.001$ ), $\mathrm{N}$ stage (hazard ratio 2.342; $\mathrm{P}<0.001$ ) and grade (hazard ratio $1.344 ; \mathrm{P}=0.001$ ) were found to be significantly associated with overall survival. The subsequent multivariate analysis (Table 2) showed that severe PWL (hazard ratio 1.534; $\mathrm{P}=0.002$ ), $\mathrm{T}$ stage (hazard ratio 1.966; $\mathrm{P}<0.001$ ), $\mathrm{N}$ stage (hazard ratio $1.967 ; \mathrm{P}<0.001$ ) and adjuvant therapy (hazard ratio $0.826 ; \mathrm{P}=0.035$ ) were independent prognostic factors for elderly patients with ESCC.

\section{Discussion}

Several studies have shown that PWL is associated with worse prognosis in patients with various types of cancer $(8,15,16)$. Due to reduced food intake with the presence 
Table 1 Demographic and clinicopathologic characteristics

\begin{tabular}{|c|c|c|c|c|c|}
\hline \multirow[b]{2}{*}{ Variables } & \multicolumn{3}{|c|}{ Weight loss } & \multicolumn{2}{|c|}{$P$ value } \\
\hline & $\begin{array}{c}\text { Normal (control) } 0 \\
(n=605)\end{array}$ & $\begin{array}{l}\text { Slightly PWL } 0-10 \% \\
\qquad(\mathrm{n}=284)\end{array}$ & $\begin{array}{l}\text { Severe PWL } \geq 10 \% \\
\qquad(n=85)\end{array}$ & $\begin{array}{l}\text { Slightly PWL vs. } \\
\text { control }\end{array}$ & $\begin{array}{l}\text { Severe PWL vs. } \\
\text { control }\end{array}$ \\
\hline Sex & & & & 0.92 & 0.34 \\
\hline Male & $486(80.33)$ & $229(80.63)$ & $72(84.71)$ & & \\
\hline \multicolumn{6}{|l|}{ Preoperative BMI } \\
\hline$\geq 18.5$ & $533(88.10)$ & $239(84.15)$ & $54(63.53)$ & 0.11 & $<0.001$ \\
\hline$<18.5$ & $72(11.90)$ & $45(15.85)$ & $31(36.47)$ & & \\
\hline \multicolumn{6}{|l|}{ Comorbidity } \\
\hline Diabetes & $16(2.64)$ & $13(4.58)$ & $3(3.53)$ & 0.13 & 0.91 \\
\hline Other cancer & $4(0.66)$ & $5(1.76)$ & 0 & 0.243 & 1.0 \\
\hline pT & & & & 0.002 & 0.000 \\
\hline $1-2$ & $243(40.17)$ & $83(29.23)$ & $14(16.47)$ & & \\
\hline $3-4$ & $362(59.83)$ & $201(70.77)$ & 71 (83.53) & & \\
\hline $\mathrm{pN}$ & & & & 0.009 & 0.004 \\
\hline 0 & $384(63.47)$ & $154(54.23)$ & $40(47.06)$ & & \\
\hline $1-3$ & $221(36.53)$ & $130(45.77)$ & 45 (52.94) & & \\
\hline pTNM & & & & 0.006 & 0.000 \\
\hline Others & 35 (5.79) & $9(3.17)$ & $3(3.53)$ & & \\
\hline Tumor location & & & & 0.444 & 0.587 \\
\hline Upper & $58(9.59)$ & $20(7.04)$ & 7 (8.24) & & \\
\hline Middle & $397(65.62)$ & $187(65.85)$ & $51(60.00)$ & & \\
\hline Lower & $145(23.97)$ & $76(26.76)$ & 26 (30.59) & & \\
\hline Cardia & $5(0.83)$ & $1(0.35)$ & $1(1.18)$ & & \\
\hline Procedure & & & & 0.350 & 0.956 \\
\hline Sweet & $500(82.64)$ & $245(86.27)$ & 72 (84.71) & & \\
\hline Ivor-Lewis & $30(4.96)$ & $13(4.58)$ & $4(4.71)$ & & \\
\hline McKeown & $55(9.09)$ & $16(5.63)$ & 7 (8.24) & & \\
\hline Other & $20(3.31)$ & $10(3.52)$ & $2(2.35)$ & & \\
\hline Adjuvant therapy & & & & 0.645 & 0.98 \\
\hline No & $412(68.10)$ & $189(66.55)$ & $58(68.24)$ & & \\
\hline Yes & $193(31.90)$ & 95 (33.45) & 27 (31.76) & & \\
\hline
\end{tabular}


of dysphagia and cancer-associated malnutrition, PWL is a common complaint for patients with ESCC. To the best of our knowledge, the number of elderly patients requiring surgical treatment for ESCC is increasing, and so there is a critical need for eligible patient selection in the elderly undergoing esophagectomy for ESCC. The presented study confirmed that $>10 \%$ PWL was an independent prognostic

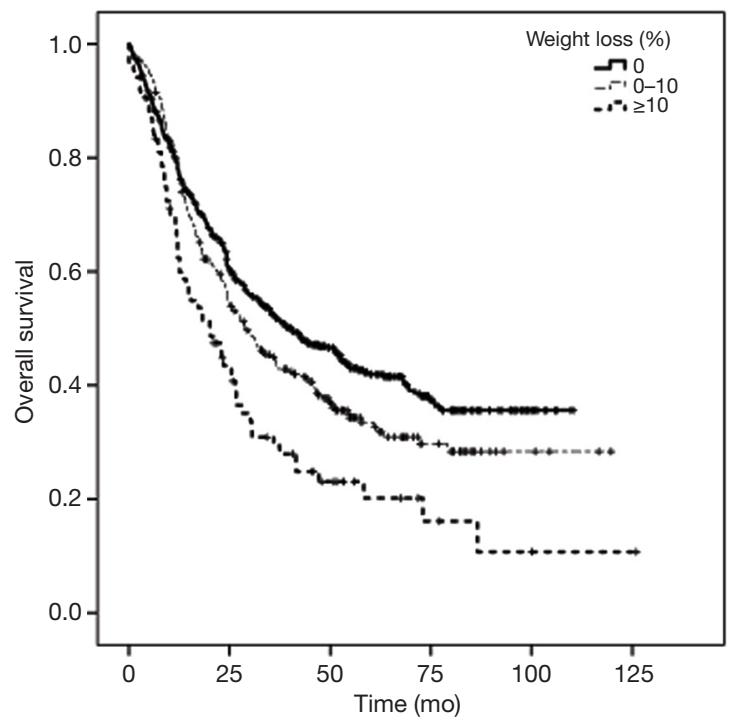

Figure 1 Kaplan-Meier curve illustrating significantly worse overall survival for patients with slight $\mathrm{PWL}$ (the thin dotted line, $\mathrm{P}=0.036$ ) and severe PWL (the thick dotted line, $\mathrm{P}<0.001$ ) compared to patients with no PWL. PEL, preoperative weight loss. factor for elderly patients undergoing surgery for ESCC. These data indicate PWL might provide important preoperative values on long-term outcome for elderly patients undergoing esophagectomy with ESCC.

Previous studies suggested that a $>10 \%$ PWL threshold represents severe nutritional risk, was associated with worse survival and an increased incidence of postoperative complications $(8,17)$. The presented study indicated that not only $>10 \%$ PWL but also $0-10 \%$ PWL were associated with decreased overall survival for elderly patients undergoing esophagectomy for ESCC. Furthermore, PWL higher than $10 \%$ was an independent risk factor of overall survival for these patients in the multivariate analysis. The definite mechanism by which PWL decreases the overall survival rate remains to be fully understood. Potentially, reduced immune function in malnourished patients might account for a reduced chance of survival (18). In addition, a low nutritional status might promote the proliferation of residual tumor cells or peripheral blood circulating tumor cells, which might also contribute to a worse survival (19).

According to available literature, about $80 \%$ of patients with advanced cancer experienced weight loss (20). Our results also indicated that patients with a PWL suffered from higher risk of lymph node involvement, local tumor invasion and advanced stage disease. Patients with advanced cancers have higher basal metabolic demands and subsequently higher energy expenditures (21), which might result in weight loss in patients with more advanced ESCC.

$\mathrm{BMI}$ has been targeted as a predictor of prognosis for

Table 2 Univariate and multivariate analyses for overall survival

\begin{tabular}{|c|c|c|c|c|c|c|}
\hline Variables & \multicolumn{3}{|c|}{ Univariate analyses } & \multicolumn{3}{|c|}{ Multivariate analyses } \\
\hline Age & 1.037 & $0.849-1.268$ & 0.720 & - & - & - \\
\hline $\operatorname{Sex}(M / F)$ & 1.388 & $1.111-1.733$ & 0.004 & 1.213 & $0.969-1.518$ & 0.092 \\
\hline Slight PWL & 1.217 & $1.014-1.460$ & 0.035 & 1.088 & $0.906-1.308$ & 0.367 \\
\hline BMI & 0.800 & $0.641-0.998$ & 0.048 & 1.042 & $0.829-1.309$ & 0.727 \\
\hline T stage & 2.418 & $1.993-2.934$ & 0.000 & 1.966 & $1.605-2.408$ & 0.000 \\
\hline $\mathrm{N}$ stage & 2.342 & $1.984-2.765$ & 0.000 & 1.967 & $1.653-2.341$ & 0.000 \\
\hline Grade & 1.344 & $1.135-1.592$ & 0.001 & 1.049 & $0.968-1.136$ & 0.244 \\
\hline
\end{tabular}

PEL, preoperative weight loss. 
patients who have undergone esophagectomy (22). Some studies have demonstrated a poor survival for underweight patients $(23,24)$. Our results revealed that BMI $<18.5$ predicted poor survival in elderly patients with ESCC in a univariate analysis. We also showed that it was not an independent factor for survival in a multivariate analysis. Due to the increased prevalence of obesity and overweight, a number of patients with esophageal cancer did not fall below their ideal weight, and BMI was normal at the time of diagnosis (25). Also, BMI changes over time for male and female (26). Therefore, we postulate that BMI alone might not an optimal indicator of long-term surgical outcome.

Both of univariate and multivariate analysis indicated PWL higher than $10 \%$ was an independent prognostic factor of overall survival for elderly patients undergoing surgery for ESCC. We hypothesize that PWL higher than $10 \%$ might be used in daily clinical practice to evaluate the nutrition status for these patients preoperatively. Our results demonstrated PWL was correlated with poor overall survival for elderly patients with ESCC, and therefore may be a promising screening tool for the identification of malnourished patients to whom perioperative nutritional support could be offered. Further study might also focus on the treatment effects of preoperative parenteral and enteral feeding for elderly patients who underwent esophagectomy for ESCC.

There exist several potential limitations of the present study. Firstly, the study was retrospective. Secondly, resulting from selection bias, only patients undergoing surgical resection were enrolled. Patients with advanced stage undergoing conservative treatment and patients with early stage disease undergoing endoscopic submucosal dissection were also not included in the analysis. In addition, neoadjuvant treatment was not routinely applied to our study population during August 2005 to April 2013, therefore only patients who underwent upfront surgical resection were included in our study.

\section{Conclusions}

For elderly patients undergoing surgery for ESCC, PWL provided prognostic value for the depth of tumor invasion, lymph node metastasis and advanced stage, and $\mathrm{PWL} \geq 10 \%$ was an independent predictor of worse overall survival.

\section{Acknowledgments}

Funding: None.

\section{Footnote}

Conflicts of Interest: All authors have completed the ICMJE uniform disclosure form (available at http://dx.doi. org/10.21037/tcr.2019.10.29). The authors have no conflicts of interest to declare.

Etbical Statement: The authors are accountable for all aspects of the work in ensuring that questions related to the accuracy or integrity of any part of the work are appropriately investigated and resolved. The study was conducted in accordance with the Declaration of Helsinki (as revised in 2013). This study was approved by West China Hospital of Sichuan University Biomedical Research Ethics Committee [No. 2016049]. Individual informed consent was waived due to the retrospective nature of the study.

Open Access Statement: This is an Open Access article distributed in accordance with the Creative Commons Attribution-NonCommercial-NoDerivs 4.0 International License (CC BY-NC-ND 4.0), which permits the noncommercial replication and distribution of the article with the strict proviso that no changes or edits are made and the original work is properly cited (including links to both the formal publication through the relevant DOI and the license). See: https://creativecommons.org/licenses/by-nc-nd/4.0/.

\section{References}

1. Zhang Y. Epidemiology of esophageal cancer. World J Gastroenterol 2013;19:5598-606.

2. Mariette C, Markar SR, Dabakuyo-Yonli TS, et al. Hybrid Minimally Invasive Esophagectomy for Esophageal Cancer. N Engl J Med 2019;380:152-62.

3. Ceccarelli G, Andolfi E, Biancafarina A, et al. Robotassisted surgery in elderly and very elderly population: our experience in oncologic and general surgery with literature review. Aging Clin Exp Res 2017;29:55-63.

4. Miller KR, Bozeman MC. Nutrition therapy issues in esophageal cancer. Curr Gastroenterol Rep 2012;14:356-66.

5. Cardinal TR, Wazlawik E, Bastos JL, et al. Standardized phase angle indicates nutritional status in hospitalized preoperative patients. Nutr Res 2010;30:594-600.

6. Martin L, Lagergren P. Long-term weight change after oesophageal cancer surgery. Br J Surg 2009;96:1308-14.

7. Van Veer H, Moons J, Darling G, et al. Validation of a new approach for mortality risk assessment in oesophagectomy for cancer based on age- and gender-corrected body mass 
index. Eur J Cardiothorac Surg 2015;48:600-7.

8. van der Schaaf MK, Tilanus HW, van Lanschot JJ, et al. The influence of preoperative weight loss on the postoperative course after esophageal cancer resection. J Thorac Cardiovasc Surg 2014;147:490-5.

9. Du XJ, Tang LL, Mao YP, et al. Value of the prognostic nutritional index and weight loss in predicting metastasis and long-term mortality in nasopharyngeal carcinoma. J Transl Med 2015;13:364.

10. Vagnildhaug OM, Blum D, Wilcock A, et al. The applicability of a weight loss grading system in cancer cachexia: a longitudinal analysis. J Cachexia Sarcopenia Muscle 2017;8:789-97.

11. Skipworth J, Foster J, Raptis D, et al. The effect of preoperative weight loss and body mass index on postoperative outcome in patients with esophagogastric carcinoma. Dis Esophagus 2009;22:559-63.

12. Sundararajan V, Hershman D, Grann VR, et al. Variations in the use of chemotherapy for elderly patients with advanced ovarian cancer: a population-based study. J Clin Oncol 2002;20:173-8.

13. Weimann A, Braga M, Harsanyi L, et al. ESPEN Guidelines on Enteral Nutrition: Surgery including organ transplantation. Clin Nutr 2006;25:224-44.

14. Yang YS, Hu WP, Ni PZ, et al. Esophageal luminal stenosis is an independent prognostic factor in esophageal squamous cell carcinoma. Oncotarget 2017;8:43397-405.

15. Qian HG, Wu LY, Li CP, et al. A novel scoring system to predict ascites development post hepatectomy for BCLC stage B hepatocellular carcinoma. Transl Cancer Res 2018;7:180-8.

16. Zuo H, Zhai L, Liu X, et al. Prognostic significance of neutrophil-lymphocyte ratio in multiple myeloma patients. Transl Cancer Res 2018;7:88-96.

17. Bozzetti F, Gianotti L, Braga M, et al. Postoperative complications in gastrointestinal cancer patients: the joint

Cite this article as: Zhang H, Yang Y, Shang Q, Li X, Wang W, Hu Y, Yuan Y, Wang Y, Chen LQ. Predictive value of preoperative weight loss on survival of elderly patients undergoing surgery for esophageal squamous cell carcinoma. Transl Cancer Res 2019;8(8):2752-2758. doi: 10.21037/ tcr.2019.10.29 role of the nutritional status and the nutritional support. Clin Nutr 2007;26:698-709.

18. Van Cutsem E, Arends J. The causes and consequences of cancer-associated malnutrition. Eur J Oncol Nurs 2005;9 Suppl 2:S51-63.

19. Nakatani M, Migita K, Matsumoto S, et al. Prognostic significance of the prognostic nutritional index in esophageal cancer patients undergoing neoadjuvant chemotherapy. Dis Esophagus 2017;30:1-7.

20. Fearon KC. Cancer cachexia: developing multimodal therapy for a multidimensional problem. Eur J Cancer 2008;44:1124-32.

21. Ravasco P, Monteiro-Grillo I, Camilo M. Colorectal cancer: intrinsic characteristics modulate cancer energy expenditure and the risk of cachexia. Cancer Invest 2007;25:308-14.

22. Zhang SS, Yang H, Luo KJ, et al. The impact of body mass index on complication and survival in resected oesophageal cancer: a clinical-based cohort and meta-analysis. Br J Cancer 2013;109:2894-903.

23. Gama RR, Song Y, Zhang Q, et al. Body mass index and prognosis in patients with head and neck cancer. Head Neck 2017;39:1226-33.

24. Masel EK, Berghoff AS, Fureder LM, et al. Decreased body mass index is associated with impaired survival in lung cancer patients with brain metastases: A retrospective analysis of 624 patients. Eur J Cancer Care (Engl) 2017;26. doi: 10.1111/ecc.12707.

25. Martin L, Lagergren J, Lindblad M, et al. Malnutrition after oesophageal cancer surgery in Sweden. Br J Surg 2007;94:1496-500.

26. Physical status: the use and interpretation of anthropometry. Report of a WHO Expert Committee. World Health Organ 1995. Available online: https://apps. who.int/iris/handle/10665/37003 